\title{
Infrared Internal Reflectance Spectroscopic Determination of Surface Functional Groups on Carbon
}

\author{
JAMES S. MATTSON AND HARRY B. MARK, JR. \\ Water Resources Science, Department of Chemistry, University of Michigan, \\ Ann Arbor, Michigan 48104 \\ Received March 5, 1969; revised April 11, 1969
}

\begin{abstract}
An internal reflectance spectrophotometric technique for the direct study of the infrared spectrum of surface functional groups on carbon is presented. The applicability and limitations of this technique are discussed. The spectra obtained for three different types of carbon; i.e., graphite, carbon black, and activated carbon, are presented. On the basis of interpretation of the spectra for these systems, it is suggested that the predominant surface functional groups on activated carbon are oxygen-containing carbonyl groups. The relative abundance of all oxygen-containing functional groups was found to be a function of both the type of carbon and its pretreatment.
\end{abstract}

\section{INTRODUCTION}

Previous research efforts in the study of adsorption on carbon surfaces have been supplemented by attempts to obtain usable infrared spectra of carbon blacks, coals, activated carbon, and graphite (1-5). For several reasons these previous efforts have been unable to obtain reliable or useful data over the entire infrared region of the spectrum. It seems obvious that reliable infrared spectra would, in conjunction with indirect observations such as adsorption studies (6), help investigators correlate the nature of surface functionality with heterogeneous adsorptive characteristics and chemical reactivity of activated carbon and carbon black. Activated carbons and carbon blacks have been shown to contain oxygen as the major impurity, and quite appropriately it is the oxygen-containing functional groups which have received the largest amount of past attention. Other impurities which are also present in some carbon systems include nitrogen, sulfur, hydrogen and ash. Nitrogen and sulfur contents are generally orders of magnitude less than that of oxygen ${ }^{1}$ (depending on the starting material), and are not considered to be a major factor in adsorption processes. The hydrogen is thought to be significant only when it is associated with the oxygen in surface functional groups. Ash is usually introduced in the manufacturing processes, but its presence appears to be of little significance in the adsorption properties of carbon, and its presence can be ignored for the present purposes.

Many investigators $(3,7,8)$ have shown that oxygen functional groups on active carbon and carbon black strongly influence the adsorptive behavior of the carbon, and it is hoped that the present work will provide a valuable tool in the positive identification of these functional groups. Two of the questions to be answered are: (i) what types of oxygen containing functional groups are present and (ii), which groups partici-

I On the basis of elemental analyses obtained by the authors from Spang Microanalytical Laboratories, or from the suppliers of sample materials, West Virginia Pulp \& Paper Co., Phillips Petroleum Co., and Ultra Carbon Corporation. 
pate in the adsorption of organic compounds from solution? A study of the interactions of the solute with oxygen functional groups on active carbon is presented in another paper (8). It is the purpose of this paper to describe in detail the experimental parameters of importance in employing internal reflectance spectroscopy for the study of surface functional groups, and to present the results of investigations of various types of carbons; active carbon, carbon black, and graphite.

Active carbons have been classified in several ways, and one of these classification schemes, Steenberg's (9) concept of " $\mathrm{H}$ " and " $L$ " carbons, which was used by Garten et al. (3, 10-12), will be mentioned here. They $(3,9,11,12)$ referred to those carbons which adsorbed considerable quantities of base as " $\mathrm{H}$ " carbons, and "L" carbons as those adsorbing similar quantities of acid (10). For the activated sugar carbons employed by Garten et al., this classification scheme has a dividing point at an activation temperature of ca. $400^{\circ} \mathrm{C}$, those activated above this temperature falling in the " $\mathrm{H}$ " classification, those below in the "L" classification.

The theory put forth by Garten et al. (3,10-12) concerning the structure of active carbon suggests that "L" carbon consists primarily of broken and crosslinked graphitelike planes, thus consisting of large planes of fused aromatic rings crosslinked by ether groups, with phenolic hydroxyl groups present at the broken edges of the fused ring systems. The presence of phenolic hydroxyl groups was inferred (3) from the fact that the titration curve of an " $L$ " carbon resembled that of a typical phenol, having a $p K_{a}$ greater than five. In order to account for the change to " $\mathrm{H}$ " behavior upon oxidation above $400^{\circ} \mathrm{C}$, they $(3,10-12)$ suggested that the ether crosslinkages are broken, and that the ether oxygens, along with the phenolic - $\mathrm{OH}$ groups, are oxidized to form quinoid structures. There is considerable evidence for the presence of car- bonyl groups on active carbon, and it is quite likely that these groups are located at the edges of the basal planes $(7,8,13,14)$. As there is no direct evidence for phenolic hydroxyl groups in "L" carbons, or quinoid structures in "H" carbons, the problem is not well resolved. Garten and Weiss (10) supported their quinone-hydroquinone argument with previous studies which indicated that some carbons chemisorb $\mathrm{Br}_{2}$, presumably (10) indicating the presence of $\mathrm{C}=\mathrm{C}$ double bonds, and that some active carbons appear to have paramagnetic character as they have been used to stabilize enzyme reaction intermediates; such stabilization being explained on the basis of a semiquinone structure on the surface of the carbon (10). Bromine uptake itself, however, is not necessarily indicative of double bond $\mathrm{C}=\mathrm{C}$ character, as will be shown below.

It is interesting to note that the presence of significant amounts of quinoid oxygen, as suggested by Garten and Weiss $(3,12)$, would force the basal ring systems into a massive fused cyclohexadiene ring system. A much more random orientation, more like a giant polymer structure, which might still contain small amounts of quinoid oxygen, is consistent with what is known $(8,14)$ about the behavior and structure of active carbons with respect to their oxygen-containing groups.

Further discussions regarding oxygencontaining groups on active carbon concern the possible presence of cyclic ester structures. It was suggested that two types of cyclic esters were responsible for some of the carbon activity; one a fluorescein-like lactone ( $f$-lactone), the other a normal lactone ( $n$-lactone) (3). The basis for this suggestion included the appearance of an absorption band at $1760 \mathrm{~cm}^{-1}$ in the transmission infrared spectrum of "Carbolac 1 ", which is a carbon black. These investigators (3) were not, however, able to obtain reliable spectra over much of the infrared region and were forced to confine their studies to the $2000-1500 \mathrm{~cm}^{-1}$ region. It is 
important to note that Garten et al. (3) also observed in this region a peak at 1600 $\mathrm{cm}^{-1}$ which appeared to correspond to the $1600 \mathrm{~cm}^{-1}$ peak observed by other investigators $(1,2,14)$. Some investigators (1-3) proposed that the $1600 \mathrm{~cm}^{-1}$ peak may be due to "... aromatic $\mathrm{C}=\mathrm{C}$ bonds or of carbonyl groups chelated to phenolic hydroxyl groups $(1,2)$." It is doubtful whether this speculation in any way supports the suggestion that phenolic hydroxyl groups are present, and from the nature of the spectroscopic arguments presented by Garten et al. (3) it is difficult to determine whether specific assignments of the 1600 and $1760 \mathrm{~cm}^{-1}$ bands are even possible without the necessary related spectra in the $3800-3300 \mathrm{~cm}^{-1} \mathrm{O}-\mathrm{H}$ stretching region.

Coughlin and Ezra (7) and Boehm (13) have characterized, by neutralization of carbon with four bases of different strengths, surface oxygen groups as being comprised of four main groups: (i) a strongly acidic carboxylic acid, (ii) a more weakly acidic carboxylic acid, (iii) a phenolic hydroxyl group, and (iv) a carbonyl group. This technique, while illustrative of the kinds of oxygen groups likely to be found on a carbon surface, is only an indirect determination, and it is reasonable to expect that a very nearly continuous series of oxygen groups of varying acidity would be formed on the carbon surface, considering the heterogeneity of possible environments for surface oxygen to be involved in.

As the above assignments of functional groups are often conflicting, and are based on bulk solution adsorption or titration measurements and limited transmission infrared data, it was felt that a more reliable spectroscopic method of determining functional groups on carbon was necessary to elucidate adsorption mechanisms (14). This paper describes the experimental parameters involved in the application of internal reflectance spectroscopy (IRS) to carbon functional group assignment, as well as the interaction of the evanescent wave in IRS with powdered samples with high extinction coefficients. The scope of applicability and the limitations of IRS for this type of investigation are discussed. A subsequent paper deals with the use of IRS data along with adsorption data for the explanation of the mechanism for adsorption of phenols on active carbon (8).

\section{EXPERIMENTAL}

In the case of bulk carbons, with a typical extinction coefficient ${ }^{2}$ of about 0.7 , the incident beam in a transmission spectrum arrangement will decay to ca. $1 \%$ of its original intensity after passing through a particle only $3.7 \mu$ thick (14). As a net result of scattering and large extinction coefficients, attempts to obtain transmission spectra of absorbing powders have resulted in a combination of transmission and reflection effects known as a diffuse reflectance spectrum. Diffuse reflectance spectra and their complications have been treated by Wendlandt and Hecht (15). These complications explain why Garten et al. (3) were unable to obtain spectra in any region but $2000-1500 \mathrm{~cm}^{-1}$, due to the difficulties experienced in trying to adequately compensate for losses in the sample beam. It also must be recognized that diffuse reflectance spectra are difficult to interpret accurately, since they will show the influence of bulk characteristics to some degree, and of external reflectance spectra of the surface to some extent, rather than selectively sample one or the other (15). Through the use of internal reflection spectroscopic techniques, these problems are eliminated; however, certain new complications then arise which must be taken into account before using IRS in the study of surfaces of absorbing powders. It has been demon-

\footnotetext{
${ }^{2}$ Foster and Howarth (17) have shown that carbons and graphite have extinction coefficients between 0.4 and 1.4 over the infrared region from 1 to $10 \mu$. A similar high extinction coefficient for graphite was obtained by Ergun (5) and Yasinski and Ergun (4) over the visible and near-infrared regions.
} 
strated previously that internal reflection spectroscopy can be applied to the study of powders $(14,16)$. While transmission and diffuse reflectance spectra are affected primarily by sample size and extinction coefficient, IRS has several more variables involved which can affect the contrast of a spectrum. These are discussed fully by Harrick (16).

The effect of depth of penetration ${ }^{3}$ (16) is extremely important in the IRS spectra of powders. When the rarer phase includes a powdered sample, it becomes a complex mixture of air and irregularly shaped absorbing particles. If one were able to obtain a spectrum of a homogeneous sample in optical contact with the IRS crystal, one would obtain high-contrast spectra with a minimum of reflections, owing to the magnitude of the interaction at the crystal-sample interface. With powders in a supporting medium making up the rarer phase, the interaction at the air-particle interface is generally much smaller than that for homogeneous samples, ${ }^{4}$ and the resulting reflectance losses are much smaller. This is described by Harrick (16) as the result of the packing characteristics of the powder.

In internal reflection spectroscopy, it is customary to use a non-absorbing $(k=0)$ material as phase 1 , the internal reflection element. In the infrared region, KRS-5,

\footnotetext{
The computer program used to calculate the $E$-fields, depths of penetrations, and reflectances (Fig. 1, as well as textual material) in this paper, according to equations 1-57 of Hansen's paper (18), has been made available to anyone through the Perkin-Elmer exchange library. Interested persons may obtain copies by requesting the program by H. C. MacDonald and J. S. Mattson from the Perkin Elmer Program Exchange Library, Perkin-Elmer Corp., Norwalk, Conn.

4 The equations for the interaction of totally reflected light with particulate samples have been derived by James S. Mattson, H. C. MacDonald, H. B. Mark, Jr., C. E. Schutt, Department of Chemistry, University of Michigan; "Reflection of a Finite Beam in IRS," and "Interaction of the Evaneseent Wave in IRS with Spherical Particles." (Both papers in preparation)
}

$\mathrm{AgCl}$ and $\mathrm{AgBr}$ fit this description. These materials are used extensively in IRS since they are transparent over the entire infrared region (16). These materials have relatively low refractive indices, however, and for carbon powders with refractive indices anywhere from 1.6 to 4 (17), such low refractive index internal reflection elements (2.0-2.4) prove unsuitable, as they preclude internal reflection for some samples over large regions of the infrared spectrum. Silicon $(n=3.5)$ and germanium $(n=4)$ would be the best IRE choices for use with carbon powders. Germanium has lattice bands starting at about $12 \mu$, but by using a carefully matched pair of Ge crystals, reliable spectra can be obtained from 2.5 to $15 \mu$ (4000-667 $\mathrm{cm}^{-1}$ ). It is quite possible that Ge crystals in this double beam arrangement could be used out to 25 or $30 \mu$, but the energy losses in both beams due to absorption by the Ge are so high beyond $15 \mu$ that the spectrophotometer response becomes too slow for satisfactory use. It is also very difficult to optically balance a pair of $\mathrm{Ge}$ crystals in the high absorption region beyond $12 \mu$. Silicon IRE crystals have not been used because of two reasons: (i) Si probably could not be used much beyond $10 \mu$ because of lattice vibration bands similar to those in $\mathrm{Ge}$, and (ii) $\mathrm{Si}$ has a refractive index of 3.5 , less than the Ge refractive index of 4 , making the Ge the more versatile IRE in that it can assure total reflection even for graphite $\left(n_{\text {graphite }}=3.5\right.$ at $\left.\lambda=8 \mu\right)$ (17).

For these studies, several different IRS geometries have been employed, varying the IRE's, the angle of incidence, and the number of reflections. Some of the spectra were obtained with germanium, varying the angle of incidence between 45 and $60^{\circ}$, and KRS-5 was used between 50 and $60^{\circ}$. (In each case, the pertinent data is listed in the figure caption.) A pair of Wilks Scientific Model 9 IRS attachments (Wilks Scientific Corp., South Norwalk, Conn.) were used in a Perkin-Elmer Model 621 
infrared spectrophotometer. Using the Wilks Model 9 IRS accessories, the Wilks MIR-29 powder sample holders were found to be the best holders available, because the crystal is held firmly and reproducibly in place in the light beam. With the MIR-29's it is possible to first obtain a baseline spectrum, remove the sample holder from the spectrometer, fill the powder holder, and replace the crystal-sample holder in the instrument without disturbing the optical arrangement. One can then run a spectrum of the powder without losing the optical alignment of the mirror system of the Model 9.

The problems associated specifically with carbon powders are all related to the fact that the samples exhibit a high extinction coefficient as well as a high refractive index over the entire infrared region of the spectrum. Table I gives representative optical parameters for graphite and carbon black (17).

From experimental studies (14) of active carbons, it appears that the $n$ and $k$ of active carbon are in the neighborhood of the values presented in Table $I$ for carbon black. These values generally increase with $\lambda$ for carbon; $2.0<n<2.4 ; 0.7<k<1.0$ for $2.5 \mu<\lambda<10 \mu$ (17). The optical constants (17) indicate that graphite $(3.0<n k<5.0)$, and active carbon $(1.4<n k<2.4)$, will be highly absorbing samples over the entire IR region. For an absorption band of a typical organic compound, the product $n k$ is zero in the neigh-

\section{TABLE I}

$n$ AND $k$ for Graphite and CARBoN BLACK, at $5 \mu$, FRom Foster and Howarth (17)

\begin{tabular}{lll}
\hline \multicolumn{2}{c}{ Refractive index, $n$} & $\begin{array}{c}\text { Extinction } \\
\text { coefficient, } k\end{array}$ \\
\hline Graphite $^{a}$ & 3.0 & 1.3 \\
Carbon black $^{b}$ & 2.25 & 0.78 \\
\hline
\end{tabular}

a Sample 4 of Foster and Howarth, a polycrystalline graphite.

${ }^{b}$ Sample 5 of Foster and Howarth, a black from natural gas. borhood of the peak, and rises to some small value at the maximum. For $p$-nitrophenol, for instance, in the IRS spectrum presented in another paper (8), the $\mathrm{C}-\mathrm{O}$ vibration of the phenolic hydroxyl group shows a maximum $n k$ of about 0.037 .

In order to observe such a vibrational band due to a surface functional group on a highly absorbing substrate, signal amplification must be employed to see a maximum difference in reflectance of about $1 \% \mathrm{R}$ (14), as the surface region is thin and the evanescent wave penetrates a considerable distance into the bulk material. The depth of penetration into a sample with $n=2.23$, $k=0.72$, will be $0.02851 \lambda$. This bulk absorption is the main factor in selection of IRS geometry and crystal arrangements.

Figure 1 shows the effect of bulk absorption at different angles of incidence for a homogeneous sample, with $n_{2}=2.25$, $k_{2}=0.78$, and $\lambda_{0}=5 \mu$, for four different IRS crystal materials; germanium $(n=4)$, gallium arsenide $(n=3.3)$, silicon $(n=$ $3.5)$, and KRS-5 $(n=2.4)$. For a single reflection by a 50:50 mixture of TM and TE polarizations, it can be seen that the bulk absorption losses can be reduced by raising the refractive index of the IRS crystal, or by increasing the angle of incidence, or both. Unfortunately, the bulk extinction coefficients of carbon samples are so high that the angles of incidence required to minimize bulk absorption (losses of $<$ $1-2 \%)$ are so high, in fact closely approaching grazing incidence, that they are not feasible for normal internal reflection techniques. It is not desirable to reduce the number of reflections below five or six, and in order to obtain multiple reflection spectra with IRS crystals that are thick enough ( $\geqq 1 \mathrm{~mm}$ ) to handle, an angle much above $60-70^{\circ}$ is just not easily obtained.

As the angle of incidence and the refractive index of the crystal are increased, there is also a corresponding loss in sensitivity in the interaction with the surface functional groups., This is because the 


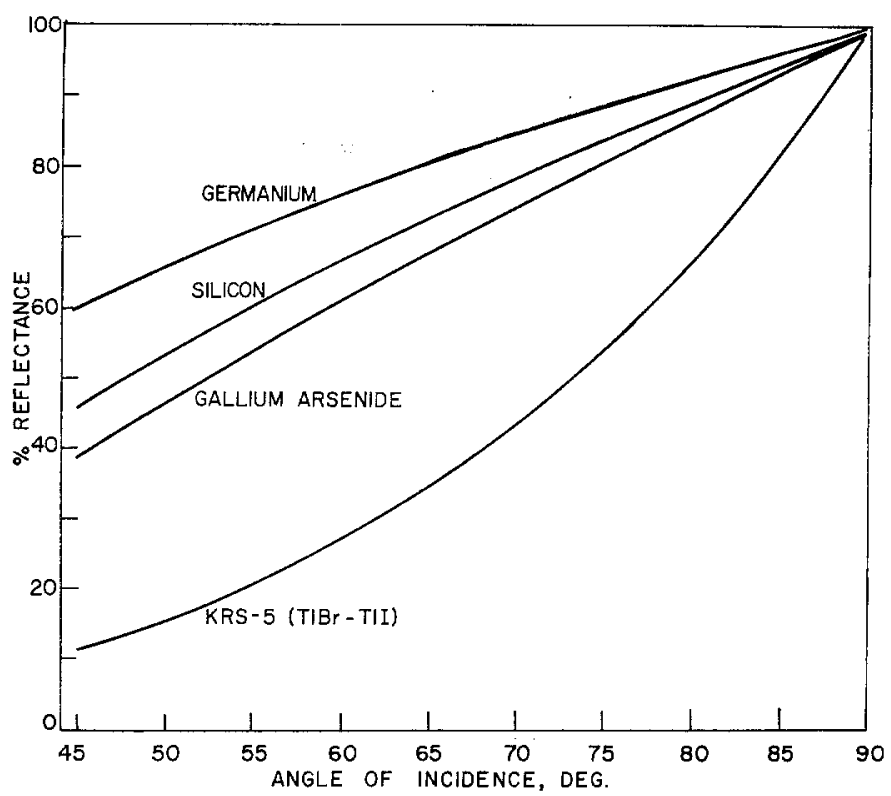

FIG. 1. IRE selection. The selections of the internal reflection element and angle of incidence are based upon the ratio of surface functional group absorption to bulk absorption. The bulk absorption for a single reflection from a homogeneous sample $(n=2.0, k=0.66, \lambda=5 \mu)$ for four different IRE materials, and $\theta_{\text {ineidence }}$ from 45 to $90^{\circ}$ is shown above.

intensity of the $E$-fields at the interface decrease rapidly with increasing angle of incidence (16). It is thus necessary to seek an alternative solution, either in the form of high electronic signal amplification, requiring the most stable of commercially available spectrophotometers, or by optically balancing out the reflectance losses caused by the bulk absorption of the substrate. An earlier paper (14) described initial experiments where graphite was employed as the optical reference material. The optical constants of graphite and active carbon ${ }^{2}$ do not, however, match well enough for graphite to make a very good reference. The ideal reference would be material identical to the sample, but with all of the surface functional groups "stripped off." This, however, is not feasible, and as yet a good reference material has not been found for these studies. Thus, whenever a reference is called for, graphite is used as it is the best available. The graphite has also been shown to contain surface oxygen groups, which serves to further complicate the problem. The graphite employed is a sub-1 $\mu$ particle size, spectroscopically pure material (UCP-1, Ultra Carbon Corp., Bay City, Michigan; prepared specially without exposure to $\mathrm{N}_{2}$ in heating cycle).

For uncompensated IRS measurements, either germanium or KRS-5 can be used as the IRE, depending on the region of the spectrum to be investigated, amplification desired, sample used, etc. Germanium presents a special problem in these high amplification applications. It is difficult to keep germanium surfaces free from adsorbed organics or surface oxides (14). It is necessary to check the optical baseline of each crystal often, looking for evidence of contamination in the 3000-2800 and 1800-1000 $\mathrm{cm}^{-1}$ regions. It is necessary to clean the crystals if contamination shows up at $5 \times$ scale expansion in the latter region. This cleaning is accomplished by repolishing the crystal using a slurry of $\$ 600 \mathrm{SiC}$ in methanol on a polishing cloth (A. B. 
Microcloth, available in 10 - and 12 -in. circles from Scientific Products, Detroit, Michigan) stretched on a glass plate and held in place with a large hose clamp. The crystal is handled with rubber finger cots and rubbed in a figure-8 motion over the $\mathrm{SiC} 20-30$ times, then moved to a dry portion of the polishing cloth and polished by rubbing 20-30 more times. This procedure is repeated as long as is necessary to obtain a "clean" $5 \times$ optical baseline, without attempting to remove all of the $\mathrm{C}-\mathrm{H}$ contamination in the $3000-2800 \mathrm{~cm}^{-1}$ region. The crystals can be washed with almost any solvent, even mild acid or alkali, without damaging the reflecting surface.

The number of reflections to be employed for best results is something which can be determined only on a trial-and-error basis for each sample. It is best to begin with a high angle of incidence and only one side of the crystal covered with powder, and then adding powder to the other side and decreasing the angle of incidence for subse-

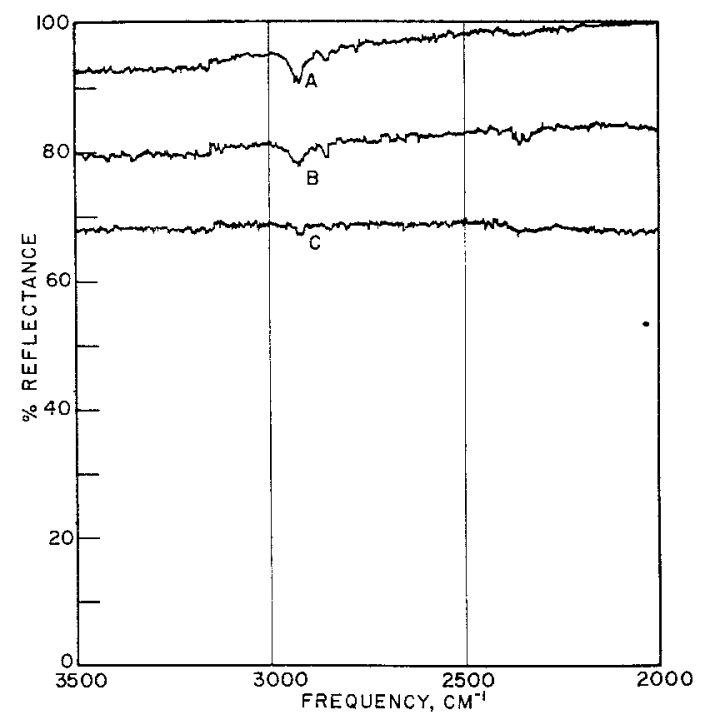

FIG. 2. Disappearance of contaminant bands. By placing an absorbing sample in contact with the IRS crystal, small absorption bands due to hydrocarbons adsorbed on the crystal can be seen to vanish. $A$ is the blank spectrum, $B$ has one side covered with carbon, and $C$ was run with both sides of the crystal covered. quent runs until the desired contrast is obtained.

One of the effects of increasing the amount of powder, or decreasing the angle of incidence is that the surface coverage of the powder itself increases the quality of the spectrum obtained by decreasing the size of the peaks contributed by contaminants on the crystal. This is shown in Fig. 2, where spectrum $\mathrm{A}$ is the crystal baseline, showing the $\mathrm{C}-\mathrm{H}$ peaks of organic material adsorbed on the Ge, B shows the peak decreasing when one side of the crystal is covered with carbon, and $\mathrm{C}$ shows that the peak all but disappears when both sides of the crystal are covered with carbon. This is as predicted theoretically by the IRS equations of Hansen $(18)^{3,4}$.

\section{RESULTS AND DISCUSSION}

Figure 3 shows the IRS spectrum of $1-\mu$ particle size graphite taken with a double beam arrangement on germanium (with just a $60^{\circ}, 1-\mathrm{mm}$ thick, 52.5 -mm-long germanium crystal in the reference beam). The number of reflections used was ca. 25 . From Fig. 3 , it can be seen that the graphite shows no distinguishing absorption bands at the normal scale of $1 \times$, although it shows strong absorption throughout the entire 2.5-15 $\mu\left(4000-667 \mathrm{~cm}^{-1}\right)$ range. Amplification of this spectrum showed that one broad absorption band was present in the $\mathrm{C}=\mathrm{O}$ region of the spectrum. This region is shown in Fig. 4, which is a 5- and $10 \times$ scale expansion of the $2000-1000 \mathrm{~cm}^{-1}$ region. In both of the expanded seale spectra of Figure 4, an intense band is observed in the 1800 $\mathrm{cm}^{-1}$ region, and another, smaller band is observed at $1475 \mathrm{~cm}^{-1}$. The band at 1800 $\mathrm{cm}^{-1}$ is abnormally broad, covering $100-200$ $\mathrm{cm}^{-1}$.

Because of the low oxygen content, about $1 \mathrm{ppm},{ }^{5}$ of the graphite it seems surprising at first that an absorption band should be observed in the $\mathrm{C}=\mathrm{O}$ region, however,

\footnotetext{
${ }^{5}$ Carl Leistner, Ultra Carbon Corp., Bay City, Michigan, personal communication.
} 


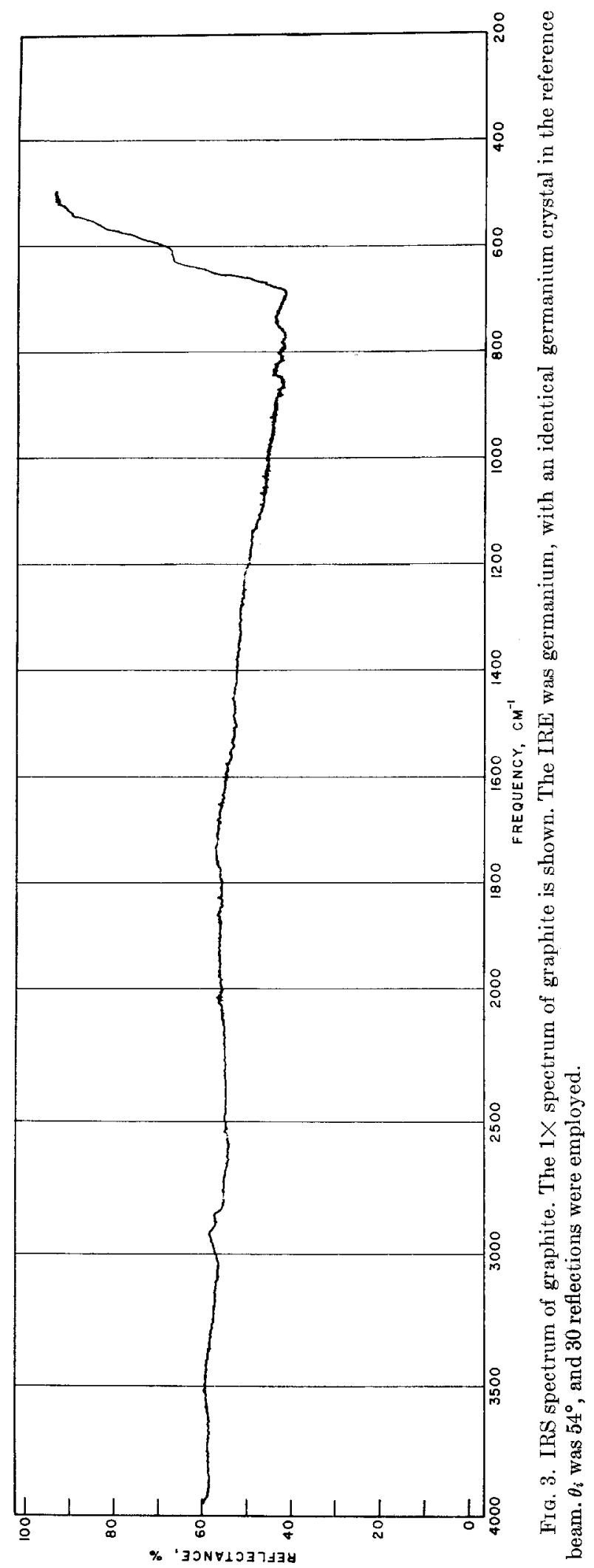

Journal of Colloid and Interface Science, Vol. 31, No. 1, September 1969 


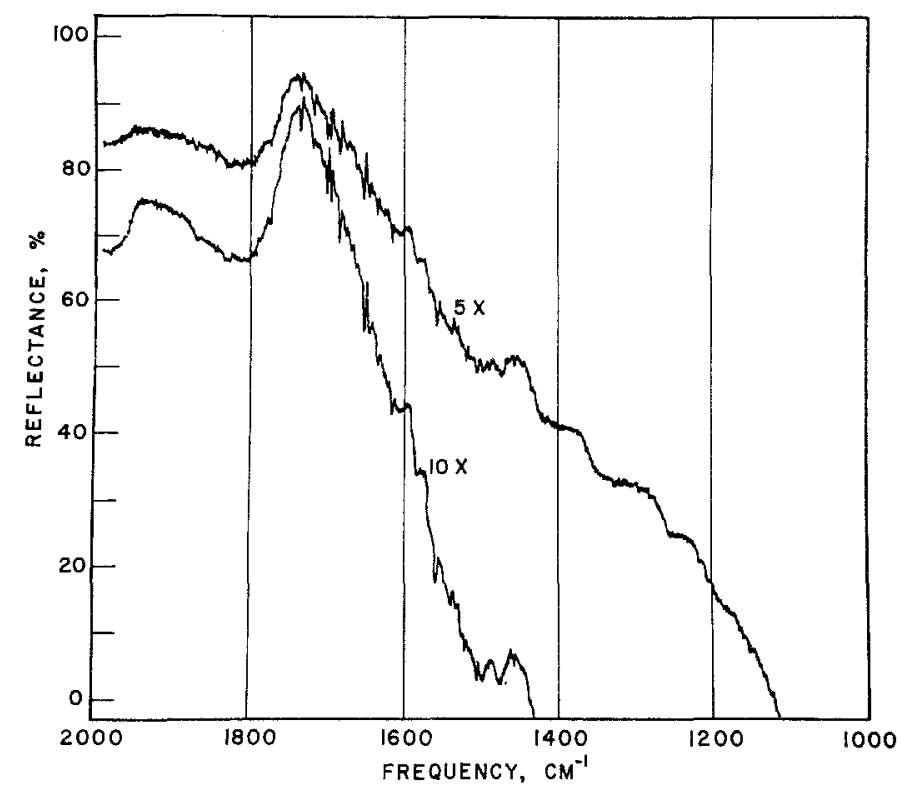

FrG. 4. $5 \times$ and $10 \times$ scale expansion of IRS spectrum of graphite. The $2000-1000 \mathrm{~cm}^{-1}$ region of the graphite spectrum of Fig. 3 is shown. This is the carbonyl oxygen region.

because of the limitation of IRS interactions to the surface regions of the sample, this could be explained on the basis of the selectivity of IRS spectra for surface structures. More on this is presented below, in relation to the active carbon spectra, where the $1800 \mathrm{~cm}^{-1}$ band also occurs.

The fact that the graphite was allowed to cool down in the manufacturing process while in limited contact with air ${ }^{5}$ implies that oxygen might have been picked up at the edges of the basal planes during the cooling procedure. The $1800 \mathrm{~cm}^{-1}$ band is quite broad, as shown in Fig. 4, which might be expected for functional groups placed in a continuous spectrum of energetically dissimilar environments. From the position of the peak, relative to comparable absorption measurements made on solutions, the $\mathrm{C}=\mathrm{O}$ groups would have to be in a strained system, most likely that of a cyclic acid anhydride. It would not be totally unexpected to find that the edges of graphitic planes would contain a large amount of broken rings which, upon oxidation, would give adjacent carboxylic acid groups. In the high temperatures encountered in the manufacturing process, the adjacent carboxylic acid groups would lose water to form the cyclic acid anhydride. However, this interpretation is far from conclusive as germanium oxides also absorb in this region, and it is found that total compensation in the dual beam arrangement is never achieved at such high amplification. Figure 4 shows that the powdered graphite bulk absorption is increasing throughout this region. In combination with uncompensated Ge-oxide absorption, this could give rise to a broad band such as that observed around $1800 \mathrm{~cm}^{-1}$.

The $1475 \mathrm{~cm}^{-1}$ band of graphite could be due to an aromatic ring vibration band, usually considerably weaker than $\mathrm{C}=\mathrm{O}$ bands. Because of the nature of the graphitic structure, however, the fact that the $\mathbf{1 4 7 5}$ $\mathrm{cm}^{-1}$ band appears to be relatively strong is not contradictory to the conclusion that it is due to the aromatic ring structure. This band is out of the carbon-oxygen region, and is difficult to identify at this time. It must be noted at this point that no phenolic, alcoholic, water or other $-\mathrm{OH}$ bands were observed in the graphite spectrum.

The spectrum of active carbon, $10 \times$ 
scale expansion (Nuchar C-1000, West Virginia Pulp \& Paper Co.) is shown in Fig. 5 , and the spectrum is strikingly similar to graphite, with broad absorption bands at $1800(1730-1850) \mathrm{cm}^{-1}$ and about 1475 $\mathrm{cm}^{-1}$. Unfortunately, the details of the manufacturing process for this carbon are not available (manufacturers vary in their willingness to cooperate in this respect), and it is more difficult to interpret spectra of this material. However, this sample was heated to $200^{\circ} \mathrm{C}$ for several days and allowed to cool in a desiccator, under vacuum, before the spectra were taken. This procedure is used to drive off the tightly bound water (not $\mathrm{H}_{2} \mathrm{O}_{2}$, misprinted in (14)) found on this series of active carbons, and this drying procedure would be sufficient to cyclize adjacent carboxylic acid groups to form the cyclic acid anhydride, as was suggested for graphite.

The spectrum shown in Fig. 6 was obtained by placing the graphite in the reference beam and the active carbon in the sample beam. The disparity in optical

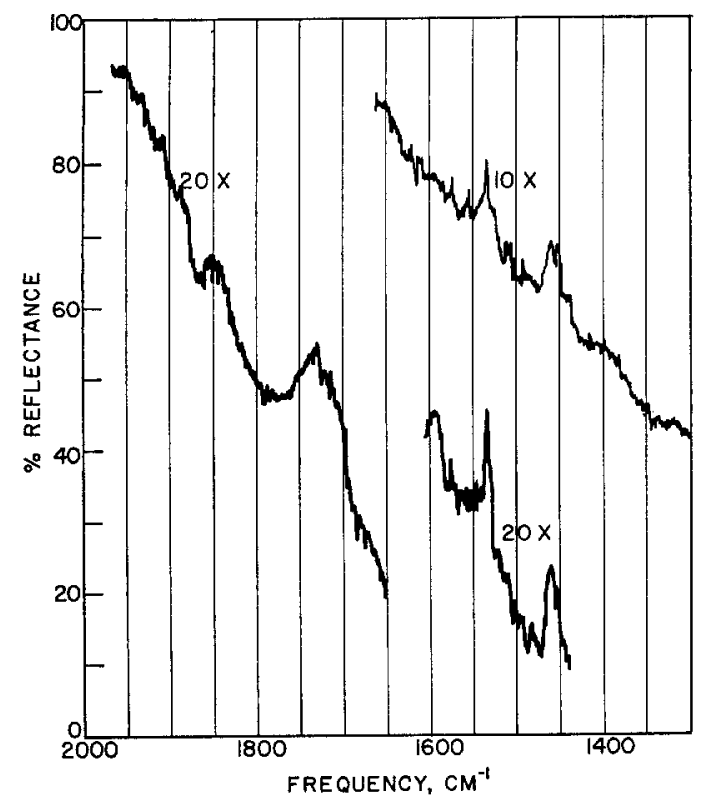

FIG. 5. 10× Expanded scale IRS spectrum of active carbon. The oxygen region is shown for an active carbon sample. The geometry is the same as that for Fig. 3, the graphite spectrum. constants is responsible for the sloping baseline in Fig. 6 (14). This Figure clearly shows that the $1800 \mathrm{~cm}^{-1}$ band is almost perfectly balanced out by the use of graphite as a reference. There is a sharp rise in the spectrum at $1775 \mathrm{~cm}^{-1}$ which is due to uncompensated germanium oxides. The fact that the broad band at $1800 \mathrm{~cm}^{-1}$ has been balanced out tends to lend support to the argument that some oxygen-containing functional group on the carbon (and graphite) is absorbing in this region. That the concentration of oxygen-containing groups on the surface of graphite is about the same as on active carbon is at first surprising, however, it must be recognized that the external surface areas of the samples, determined by particle size and shape, are about equal, and that the tremendous amount of internal surface area of the active carbon, which accounts for about $99 \%$ of its surface area (8), is not accessable to the evanescent wave in IRS because of the very limited

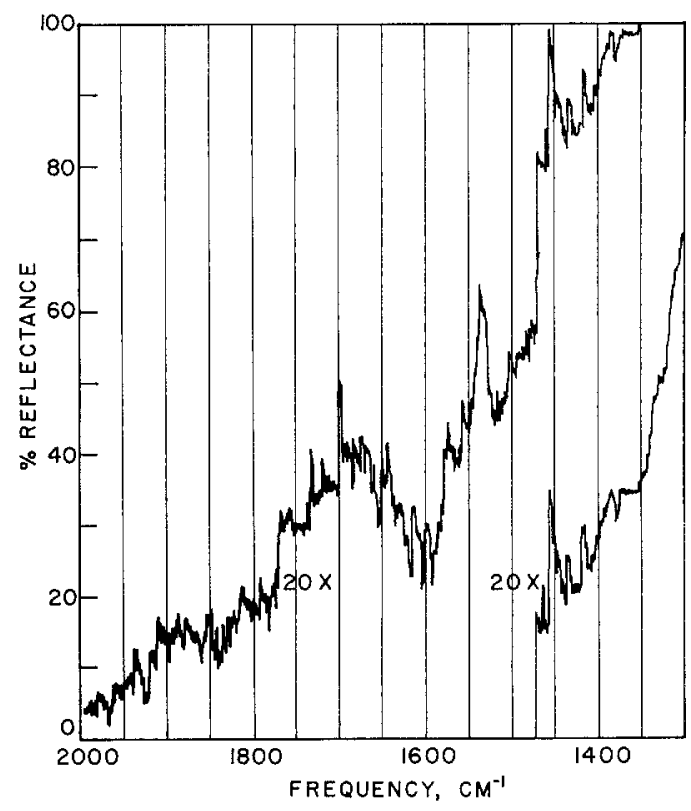

Fig. 6. 10× Expanded scale spectrum, active carbon vs. graphite. The oxygen region of the "compensated" spectrum is shown. The same IRS geometry as in Figs. 3-5 is used, but with a graphite sample in the reference beam used to counteract some of the bulk absorptinn. 
depths of penetration encountered. Therefore, the concentration of oxygen on the external surfaces in both samples could be equal even if the total $N_{2}$ surface area varied by three orders of magnitude, this by assuming that the total oxygen concentrations are between three and four orders of magnitude apart. As it was pointed out before (8), the $\mathrm{O}$ content of the $\mathrm{C}-1000$ is in the $3-5 \%$ range, and ${ }^{5}$ that of the graphite is about $1 \mathrm{ppm}$; the surface areas are about (8) $1000 \mathrm{~m}^{2} / \mathrm{g}$ for the active carbon, and about $1 \mathrm{~m}^{2} / \mathrm{g}$ for the graphite.

Figure 5 also shows that the $1475 \mathrm{~cm}^{-1}$ band of the graphite is not as strong as that in the active carbon, which could indicate more "loosely attached" aromatic rings on the active carbon than on the graphite.

There is a new absorption band observed in Fig. 6 when the graphite reference is used, which was evidently obscured in the singleIRS-beam spectrum (Fig. 5). This is the broad band centered around $1600 \mathrm{~cm}^{-1}$ which appears to correspond to the one found earlier (14) and to those observed by other investigators (1-3) in their diffuse reflectance studies. This $1600 \mathrm{~cm}^{-1}$ band has been attributed $(1-3,14)$ to some sort of $\mathrm{C}=\mathrm{C}$ vibration, and in the light of the observation of a band at $1475 \mathrm{~cm}^{-1}$ in graphite and in active carbon, the $\mathrm{C}=\mathrm{C}$ designation could not be due to the graphitic structure (for the $1600 \mathrm{~cm}^{-1}$ band). The only other possible assignment for the $1600 \mathrm{~cm}^{-1}$ band is that of a ketone $\mathrm{C}=\mathrm{O}$ vibration, and this assignment is apparently supported by the adsorption studies on this active carbon (8), and by some of the previous data taken by Coughlin and Ezra ( 7 ) indicating that surface oxygens were found with a whole range of $p K_{a}$ values, including some which were no more acidic than ketone carbonyl groups. The mention by Garten et al., of $\mathrm{Br}_{2}$ uptake (10) on some carbons, as discussed in the introduction, does not necessarily indicate double bond $\mathrm{C}=\mathrm{C}$ character. In fact, it is well-known (19) that carbonyl compounds with $\alpha$-hydrogens bro- minate readily in either acidic or basic media.

Figure 7 shows the entire infrared spectrum of a carbon black (Philblack S315, Phillips Petroleum Co., Bartlesville, Okla.). This carbon black spectrum shows more detail than the graphite spectrum in Fig. 3 because (i) the IRE was KRS-5, with $\theta_{i}=54^{\circ}$, and (ii) the spectrum in Fig. 7 is at $2 \times$ scale expansion. This particular IRE selection was made possible by the use of a large particle size sample; the sample was received in pelletized form, and this made it possible to use a lower refractive index IRE because of the thick air layer through which the evanescent wave had to pass.

The as received carbon black has only one distinguishing feature, observed both in Fig. 7, and in the expanded scale spectrum in Fig. 8. This is a strong band in the carboxylic acid $\mathrm{C}=\mathrm{O}$ region, at $1700 \mathrm{~cm}^{-1}$. There is also a weaker band at $3400 \mathrm{~cm}^{-1}$, which is in the $-\mathrm{OH}$ region, but is also at exactly the right frequency to be the overtone of the band at $1700 \mathrm{~cm}^{-1}$. As this sample had not been dried or otherwise specially treated before the IRS study, assignment of the $3400 \mathrm{~cm}^{-1}$ band could be made to either a carboxylic acid $-\mathrm{OH}$ (the most likely, considering the obvious carboxylic acid band at $1700 \mathrm{~cm}^{-1}$ ), or to the overtone of the $1700 \mathrm{~cm}^{-1}$ band (the next best possibility), or to a phenolic - $\mathrm{OH}$ (the least likely), or to water (not likely because of the wrong positions and intensities of what would be the water bands at 3350 and $1650 \mathrm{~cm}^{-1}$ ). It should be pointed out that carbon black, by its very nature, would not be expected to show very pronounced graphite-like structural characteristics (17). Since this carbon black sample was not purified or dried as were the graphite and active carbon samples, and considering the fact that this carbon black gives a $\mathrm{pH}$ of 2.5 in a water slurry, the assignment of the $1700 \mathrm{~cm}^{-1}$ band to carboxylic acid seems well supported. It was 


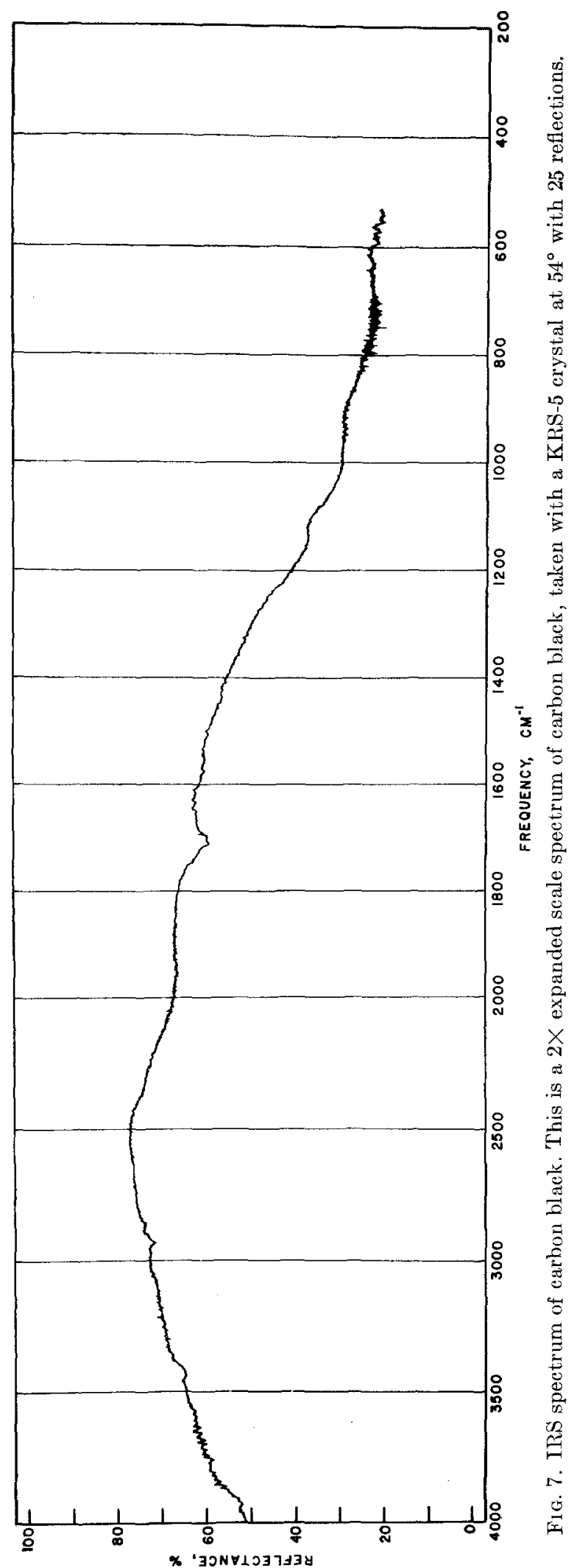

Journal of Colloid and Interface Science, Vol. 31, No. 1, September 1969 

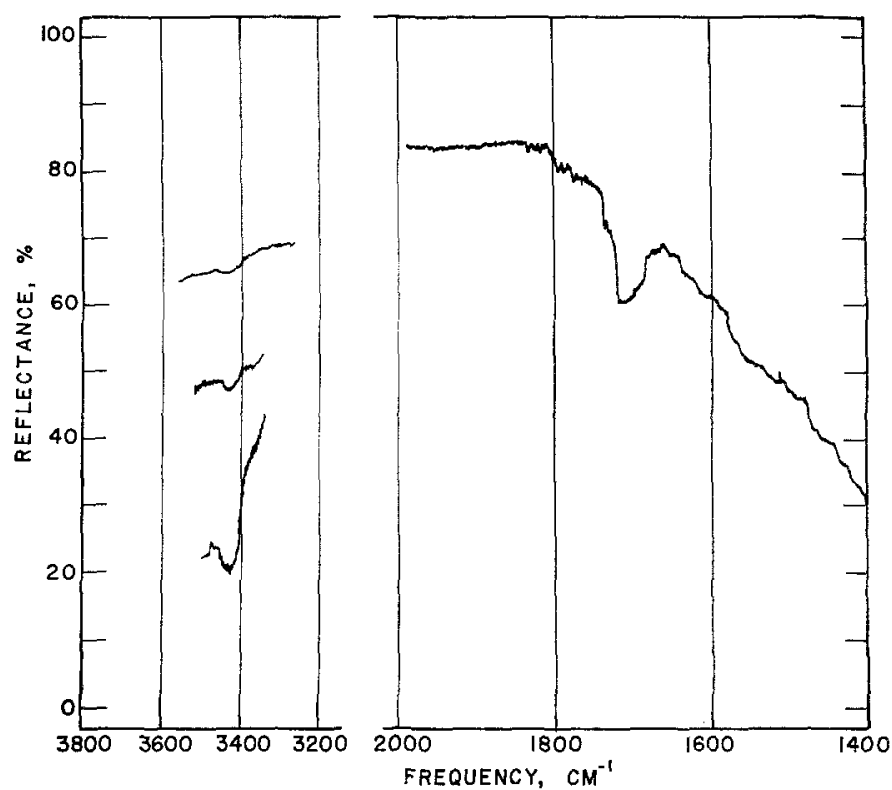

Frg. 8. $5 \times$ and $10 \times$ expanded scale spectra of carbon black. Using the same IRS geometry as Fig. 11, but expanding the regions of maximum interest.

found that on purification and drying of the carbon black sample, ${ }^{6}$ the 1700 and 3400 $\mathrm{cm}^{-1}$ bands disappeared and the $\mathrm{pH}$ of a water slurry was only 4.2. This indicates that the carboxylic acid group is present as an impurity. ${ }^{6}$

On the basis of the above experimental data and conclusions, it can be said that, at least for the limited sampling done here, the predominant oxygen-containing functional groups observed on active carbon are ketone carbonyl groups. None of the quinone groups suggested by Garten et al. $(3,10-12)$ were observed, nor were any phenolic hydroxyl groups, since the 3400 $\mathrm{cm}^{-1}$ band on carbon black has been shown to be due to an impurity.

\section{ACKNOWLEDGMENTS}

The authors would like to acknowledge the help of Andrew Loven, West Virginia Pulp \& Paper Co.,

6 The purification was carried out as follows: The sample was washed several times with hot toluene, followed by hexane, then heated at $600^{\circ} \mathrm{C}$ for $30 \mathrm{~min}$, followed by $24 \mathrm{hr}$ at $200^{\circ} \mathrm{C}$, then one week at $105^{\circ} \mathrm{C}$. It was allowed to equilibrate with the atmosphere during the cooling down to room temperature. The water slurries were made up at a concentration of $1 \mathrm{~g}$ /liter in triply distilled water.
Jay Janzen, Phillips Petroleum Co., and Carl Leistner, Ultra Carbon Corp., for providing sample materials and information. They would also like to acknowledge the invaluable cooperation of the Chemistry Department at the University of Toledo for allowing their Perkin-Elmer 621 to be used in these studies. Technical advice was obtained from N. J. Harrick of Phillips Laboratories and Paul Wilks, Jr. of Wilks Scientific, and the authors are grateful for such assistance. Paul Wilks, Jr. also contributed some of the original IRS accessories used in the authors' laboratory.

This research was supported in part by the National Science Foundation, Grant GP-9307.

This paper presented at the 43rd National Colloid Symposium, Colloid and Surface Chemistry Division, ACS, Case Western Reserve University, Cleveland, Ohio, June 23-5, 1969.

\section{REFERENCES}

1. Friedel, R. A. and Queiser, J. A., Anal. Chem. 28, 22 (1956).

2. Brown, J. K., J. Chem. Soc. (London) 1955, 744.

3. Garten, V. A., Weiss, D. E., Wiliss, J. B., Australian J. Chem. 10, 295 (1957).

4. Yasinski, J. B. AND Ergun, S., Carbon 2, 355 (1965).

5. Ergun, S., In "Chemistry and Physies of Carbon," Vol. 3, (Philip L. Walker, Ed.) pp. 45-119, Marcel Dekker, New York, 1968.

6. SNoEYınk, V. L., Ph.D. Thesis, Univ. of Michigan, 1968. 
7. Coughlin, R. W. And EzRa, F. S., Environ. Sci. Technol. 2, 291 (1968).

8. Mattson, J. S., Mark, H. B., Jr., Malbin, M. D., Weber, W. J., JR., CritTenden, J. C., J. Colloid and Interface Sci. 31, 116 (1969).

9. STEenBerg, B., "Adsorption and Exchange of Ions on Activated Charcoal," Almquist and Wilksells, Uppsala, 1944. Ph.D. Thesis, Stockholm University. (Ref. 23 of 10.)

10. Garten, V. A. And Weiss, D. E., Rev. Pure Appl. Chem. 7, 69 (1957).

11. - - A Australian J. Chem. 8, 68 (1955).

12. —, Australian J. Chem. 10, 309 (1957).
13. Bowhm, H. P., Advan. Catalysis 16, 179 (1964).

14. Mattson, J. S., Mark, H. B., JR., Weber, W. J., JR., Anal. Chem. 41, 355 (1969).

15. Wendland, W. W., and Hecht, H. G., "Reflectance Spectroscopy," pp. 46-79, Interscience, New York, 1966.

16. Harrick, N. J., "Internal Reflection Spectroscopy," Interscience, New York, 1967.

17. Foster, P. J. and Howarth, C. R., Carbon 6 , 719 (1968).

18. Hansen, W. N., J. Opt. Soc. Am. 58, 380 (1968).

19. House, H. O., "Modern Synthetic Reactions," p. 144, Benjamin, New York, 1965. 